\title{
Computation of fractional derivatives using Fourier transform and digital FIR differentiator
}

\author{
Chien-Cheng Tseng ${ }^{a}, *$ Soo-Chang Pei ${ }^{b}$, Shih-Chang Hsia ${ }^{a}$ \\ ${ }^{\mathrm{a}}$ Department of Computer and Communication Engineering, National Kaohsiung First University of Science and Technology, Kaohsiung, \\ Taiwan \\ ${ }^{\mathrm{b}}$ Department of Electrical Engineering, National Taiwan University, Taipei, Taiwan
}

\begin{abstract}
In this paper, the computation of a fractional derivative using the Fourier transform and a digital FIR differentiator is investigated. First, the Cauchy integral formula is generalized to define the fractional derivative of functions. Then the fractional differentiation property of the Fourier transform of functions is presented. Using this property, the fractional derivative of a function can be computed in the frequency domain. Next, we develop a least-squares method to design the fractional order digital differentiator. When a signal passes through the designed differentiator, the output will be its fractional derivative. One design example is included to illustrate the effectiveness of this approach. Finally, the designed fractional order differentiator is used to generate a random fractal process which is better than the process obtained by the conventional method. (C) 2000 Elsevier Science B.V. All rights reserved.
\end{abstract}

\section{Zusammenfassung}

In diesem Artikel wird die Berechnung der fraktalen Ableitung unter Verwendung der Fouriertransformation und eines digitalen, transversalen Differentiators untersucht. Zunächst wird die Formel des Cauchy-Integrals verallgemeinert, um die fraktale Ableitung von Funktionen zu definieren. Dann wird die fraktale Differenzierungseigenschaft der Fouriertransformation von Funktionen vorgestellt. Mittels dieser Eigenschaft kann die fraktale Ableitung einer Funktion im Frequenzbereich berechnet werden. Als nächstes entwickeln wir eine Kleinste-Quadrate-Methode, um den digitalen Differentiator gebrochener Ordnung zu entwerfen. Wenn ein Signal den entworfenen Differentiator durchläuft, wird das Ausgangssignal seine fraktale Ableitung sein. Ein Entwurfsbeispiel ist angegeben, um die Effektivität diese Methode zu veranschaulichen. Schließlich wird der entworfene Differentiator gebrochener Ordnung verwendet, um einen fraktalen, stochastischen Prozeß zu generieren, der besser als der Prozeß nach herkömmlicher Methode ist. (C) 2000 Elsevier Science B.V. All rights reserved.

\section{Résumé}

Dans cet article, nous investiguons le calcul d'une dérivée fractionnelle en utilisant la transformation de Fourier et un filtre FIR différentiateur. Tout d'abord, la formule de l'intégrale de Cauchy est généralisée pour définir la dérivée fractionnelle de fonctions. Ensuite la propriété de différentiation fractionnelle de la transformée de Fourier de fonctions est présentée. En utilisant cette propriété, la dérivée fractionnelle d'une fonction peut être calculée dans le domaine fréquentiel. Ensuite, nous développons une méthode des moindres carrés pour concevoir un différentiateur numérique

\footnotetext{
* Corresponding author.

E-mail address: tcc@ccms.nkfu.edu.tw (C.-C. Tseng)
} 
d'ordre fractionnel. Lorsqu'un signal passe au travers du différentiateur ainsi conçu, la sortie sera sa dérivée fractionnelle. Un exemple de conception est inclus pour illustrer l'efficacité de cette approche. Finalement, le différentiateur d'ordre fractionnel ainsi conçu est utilisé pour générer un processus fractal aléatoire meilleur que le processus obtenu par la méthode conventionnelle. (C) 2000 Elsevier Science B.V. All rights reserved.

Keywords: Fractional derivative; Fourier transform; Differentiator

\section{Introduction}

In recent years, the concept of fractional operator and measure has been investigated extensively in many engineering applications and science. Four typical examples are described as follows. The first, is fractional derivative and integral are defined by many mathematicians and applied to solve some physical problems $[9,12]$. The second, is the fractional Fourier transform has been studied in the optical community and signal processing area $[1,10,17]$. The third, is the fractional dimension is used to measure some real-world data such as coastline, clouds, dust in the air, and network of neurons in the body. The fractional dimension has been applied widely to pattern recognition and classification [2]. The last is fractional lower-order moment has been used to analyize non-Gaussian signal which is more realistic than the Gaussian model in signal processing applications [19].

In the research area of fractional calculus, the integer order $n$ of derivative $D^{n} f(x)=\mathrm{d}^{n} f(x) / \mathrm{d} x^{n}$ of function $f(x)$ is generalized to fractional order $D^{v} f(x)$, where $v$ is a non-integer. Although fractional calculus is useful in many fields of sciences and engineering, including fluid flow, automatic control, electrical networks, electromagnetic theory, and probability $[5-8,11,20]$, it has not drawn the attention of researchers in the signal processing area. The purpose of this paper is to use signal processing tools such as the Fourier transform and the digital FIR differentiator, to compute fractional derivatives of signals. Because the fractional derivative of a zero mean white noise is a fractal process called Brownian motion ( $\mathrm{fBm})$, the proposed method can be used to generate the fractal process.

Conventionally, the digital differentiator is a very useful tool to determine and estimate the time derivatives of a given signal. For example, in radar and sonar applications, the velocity and acceleration are computed from position measurements using differentiators [21]. In biomedical engineering, it is often necessary to obtain the higher-order derivatives of biomedical data, especially at lowfrequency ranges [24]. So far, several methods have been developed to design IIR and FIR digital differentiators such as the Remez exchange algorithm [14], the eigenfilter method $[15,16]$, and the weighted least-squares method $[22,23]$, etc. An excellent survey of differentiator design has been presented in a tutorial paper [4]. However, the orders of differentiators in the above applications are all integers, so it cannot be applied to compute fractional derivatives. In this paper, differentiators will be generalized to fractional order to achieve our purpose.

The paper is organized as follows. In Section 2, the Cauchy integral formula is generalized to define the fractional derivative of functions. In Section 3, the fractional differentiation property of the Fourier transform of functions is presented. Using this property, the fractional derivative of a function can be computed in the frequency domain. In Section 4, we develop a least squares method to design a fractional order digital differentiator. When a signal passes through the designed differentiator, the output will be its fractional derivative. One design example is included to illustrate the effectiveness of this approach. In Section 5, the designed fractional order differentiator is used to generate a random fractal process which is better than the process obtained by the conventional method.

\section{Definition of fractional derivatives}

In this section, a form of complex integral transformation will be used to define fractional 
derivatives. We begin with the statement of the Cauchy integral formula [18]: If $f(z)$ is singlevalued and analytic in an open region $D$ of the complex plane, and if $A$ is an open region interior to $D$ bounded by a closed smooth curve $C$, then

$f(z)=\frac{1}{2 \pi \mathrm{i}} \int_{C} \frac{f(\zeta)}{\zeta-z} \mathrm{~d} \zeta$

for any point $z$ in $A$. From (1), it follows that

$D^{n} f(z)=\frac{n !}{2 \pi \mathrm{i}} \int_{C} \frac{f(\zeta)}{(\zeta-z)^{n+1}} \mathrm{~d} \zeta$

where $n$ is an integer. If $n$ is generalized to an arbitrary order $v$, we may replace $n$ ! by gamma function $\Gamma(v+1)$ in (2) to obtain the definition of the fractional derivative as follows:

$D^{v} f(z)=\frac{\Gamma(v+1)}{2 \pi \mathrm{i}} \int_{C} \frac{f(\zeta)}{(\zeta-z)^{v+1}} \mathrm{~d} \zeta$

where the choice of contour $C$ is described in [11]. Using this definition, the fractional derivatives of exponential and trigonometric functions are given by

$D^{v} \mathrm{e}^{a z}=a^{v} \mathrm{e}^{a z}$,

$D^{v} \cos (a z)=a^{v} \cos \left(a z+\frac{\pi}{2} v\right)$

$D^{v} \sin (a z)=a^{v} \sin \left(a z+\frac{\pi}{2} v\right)$

where $z \in C, v \in R$ and $a(\neq 0)$ is a constant [11]. From this result, it is clear that when order $v$ goes from 0 to 1 , the derivative $D^{v} \sin (a z)$ goes from $\sin (a z)$ to $a \cos (a z)=a \sin (a z+\pi / 2)$ by gradually increasing amplitude from 1 to $a$ and phase from 0 to $\pi / 2$. Thus, the fractional derivative can be interpreted as a function interpolation between derivatives with integer order. In order to illustrate this claim, let us further investigate the fractional derivative of the rectangular wave $f(t)$ defined by

$f(t)= \begin{cases}V, & n T<t<\left(n+\frac{1}{2}\right) T, \\ -V, & \left(n+\frac{1}{2}\right) T<t<(n+1) T,\end{cases}$ (a)

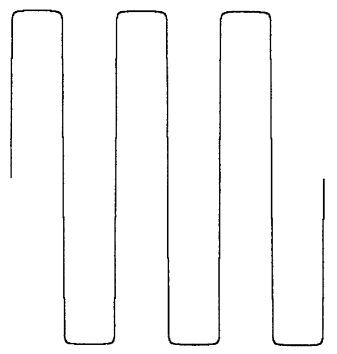

(c)

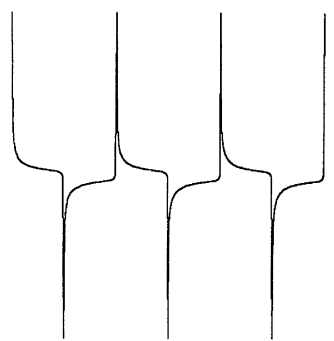

(b)

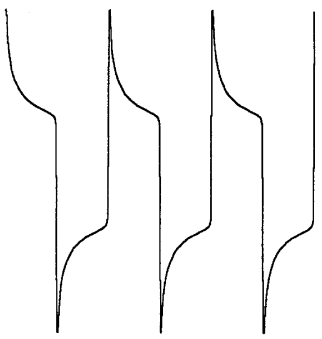

(d)

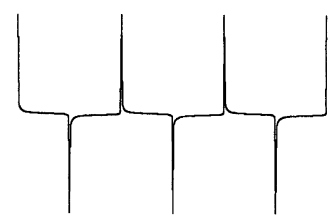

Fig. 1. The fractional derivative of rectangular wave for various order $v$. (a) $v=0$, (b) $v=0.2$, (c) $v=0.5$, (d) $v=0.7$.

where $T$ is the period. According to the Fourier series, $f(t)$ can be written as

$f(t)=\sum_{n=1, n \in \mathrm{odd}}^{\infty} \frac{4 V}{n \pi} \sin \left(\frac{2 n \pi t}{T}\right)$.

Since the differential operator is linear, the fractional derivative of the rectangular wave is given by

$D^{v} f(t)=\sum_{n=1, n \in \text { odd }}^{\infty} \frac{4 V}{n \pi}\left(\frac{2 n \pi}{T}\right)^{v} \sin \left(\frac{2 \pi n t}{T}+\frac{v \pi}{2}\right)$.

Choosing parameters $V=1$ and $T=0.01$, Fig. 1 shows the fractional derivative $D^{v} f(t)$ of the rectangular wave for various order $v$. It is clear that the waveform of the fractional derivative evolves very smoothly as order $v$ increases.

\section{Computation of fractional derivatives using the Fourier transform}

\subsection{Fourier transform of fractional derivatives}

The Fourier transform is an integral operation and defined by the following transform 
pair:

$F(\omega)=\int_{-\infty}^{\infty} f(t) \mathrm{e}^{-\mathrm{i} \omega t} \mathrm{~d} t$,

$f(t)=\frac{1}{2 \pi} \int_{-\infty}^{\infty} F(\omega) \mathrm{e}^{\mathrm{i} \omega t} \mathrm{~d} \omega$.

Applying the fractional differential operator to both sides of (8), we obtain

$$
\begin{aligned}
D^{v} f(t) & =D^{v}\left[\frac{1}{2 \pi} \int_{-\infty}^{\infty} F(\omega) \mathrm{e}^{\mathrm{i} \omega t} \mathrm{~d} \omega\right] \\
& =\frac{1}{2 \pi} \int_{-\infty}^{\infty} F(\omega)\left[D^{v} \mathrm{e}^{\mathrm{i} \omega t}\right] \mathrm{d} \omega
\end{aligned}
$$

Since $D^{v} \mathrm{e}^{a z}=a^{v} \mathrm{e}^{a z}$ holds, take $a=\mathrm{i} \omega$ and $z=t$ such that

$D^{v} \mathrm{e}^{\mathrm{i} \omega t}=(\mathrm{i} \omega)^{v} \mathrm{e}^{\mathrm{i} \omega t}$.

Substituting (10) into (9), we obtain

$D^{v} f(t)=\frac{1}{2 \pi} \int_{-\infty}^{\infty}(\mathrm{i} \omega)^{v} F(\omega) \mathrm{e}^{\mathrm{i} \omega t} \mathrm{~d} \omega$.

Thus, the Fourier transform of $v$ th-order derivative $D^{v} f(t)$ is given by $(\mathrm{i} \omega)^{v} F(\omega)$.

\subsection{Computation of fractional derivatives using the} Fourier transform

The result in (11) tells that when a signal $f(t)$ passes through a linear system with frequency response $(\mathrm{i} \omega)^{v}$, the output signal of the system will be fractional derivative $D^{v} f(t)$. Based on this fact, a procedure to compute the fractional derivative of signal $f(t)$ in frequency domain is given as follows: Step 1: Compute the Fourier transform $F(\omega)$ of signal $f(t)$.

Step 2: Compute the inverse Fourier transform of $(\mathrm{i} \omega)^{v} F(\omega)$ to get the fractional derivative $D^{v} f(t)$.

An example is used to demonstrate this method. If $f(t)$ is a sinusoidal signal denoted by $\cos \left(\omega_{0} t\right)$, its Fourier transform is given by

$$
F(\omega)=\pi \delta\left(\omega-\omega_{0}\right)+\pi \delta\left(\omega+\omega_{0}\right) .
$$

Since $\left(\mathrm{i} \omega_{0}\right)^{v}=\mathrm{e}^{\mathrm{i} v \pi / 2} \omega_{0}^{v}$ and $\left(-\mathrm{i} \omega_{0}\right)^{v}=\mathrm{e}^{-\mathrm{i} v \pi / 2} \omega_{0}^{v}$, we obtain

$$
\begin{aligned}
(\mathrm{i} \omega)^{v} F(\omega)= & \pi \omega_{0}^{v} \delta\left(\omega-\omega_{0}\right) \mathrm{e}^{\mathrm{i} v \pi / 2} \\
& +\pi \omega_{0}^{v} \delta\left(\omega+\omega_{0}\right) \mathrm{e}^{-\mathrm{i} v \pi / 2} .
\end{aligned}
$$

Taking the inverse Fourier transform, we obtain fractional derivative as follows:

$$
D^{v} f(t)=\omega_{0}^{v} \cos \left(\omega_{0} t+\frac{v \pi}{2}\right)
$$

which is consistent with the result in (4).

\subsection{Extension to the discrete time case}

So far, we have been limited to the continuous time case. Now let us extend these results to the discrete time signal case. Using the definition in the frequency domain, the procedure to determine the fractional derivative of sequence $x(n)$ is stated below:

Step 1: Find the discrete time Fourier transform of $x(n)$ by using the formula [13]:

$X(\omega)=\sum_{n=-\infty}^{\infty} x(n) \mathrm{e}^{-\mathrm{i} \omega n}$

Step 2: Compute the inverse Fourier transform of $(\mathrm{i} \omega)^{v} X(\omega)$ to obtain the fractional derivative $D^{v} x(n)$ by using the equation:

$D^{v} x(n)=\frac{1}{2 \pi} \int_{-\pi}^{\pi}(\mathrm{i} \omega)^{v} X(\omega) \mathrm{e}^{\mathrm{i} \omega n} \mathrm{~d} \omega$.

We use the same example to demonstrate this method. If $x(n)$ is a sinusoidal signal denoted by $\cos \left(\omega_{0} n\right)$, its discrete time Fourier transform is given by

$$
\begin{aligned}
X(\omega)= & \pi \sum_{k=-\infty}^{\infty}\left[\delta\left(\omega-\omega_{0}+2 \pi k\right)\right. \\
& \left.+\delta\left(\omega+\omega_{0}+2 \pi k\right)\right] .
\end{aligned}
$$

Thus, in the interval $[-\pi, \pi]$, we have

$$
\begin{aligned}
(\mathrm{i} \omega)^{v} X(\omega)= & \pi\left[\omega_{0}^{v} \delta\left(\omega-\omega_{0}\right) \mathrm{e}^{\mathrm{i} v \pi / 2}\right. \\
& \left.+\omega_{0}^{v} \delta\left(\omega+\omega_{0}\right) \mathrm{e}^{-\mathrm{i} v \pi / 2}\right] .
\end{aligned}
$$


Take the inverse Fourier transform, we obtain the fractional derivative as follows:

$D^{v} x(n)=\omega_{0}^{v} \cos \left(\omega_{0} n+\frac{v \pi}{2}\right)$.

When $x(n)$ is a general time sequence, it is difficult to use the above procedure to compute the fractional derivative of $x(n)$. However, when $x(n)$ passes through the digital filter with frequency response $(i \omega)^{v}$, the filter output will be derivative $D^{v} x(n)$. Thus, the problem of computing the fractional derivative of sequence $x(n)$ becomes one of the designs of a filter with frequency response $(i \omega)^{v}$. In next section, this topic will be addressed.

\section{Computation of fractional derivatives using the digital differentiator}

\subsection{Design of fractional digital differentiator}

In the following, we will design an FIR filter to approximate the specification (iw) ${ }^{v}$ of fractional derivative. The transfer function of a causal $\mathrm{Nth}$ order FIR filter can be represented by

$H(z)=\sum_{n=0}^{N} h(n) z^{-n}$

The frequency response of the FIR filter is given by

$H(\omega)=\boldsymbol{h}^{\mathrm{t}} \boldsymbol{e}(\omega)=\boldsymbol{e}^{\mathrm{t}}(\omega) \boldsymbol{h}$,

where vectors $\boldsymbol{h}$ and $\boldsymbol{e}(\omega)$ are

$\boldsymbol{h}=[h(0) h(1) \cdots h(N)]^{\mathrm{t}}$,

$\boldsymbol{e}(\omega)=\left[1 \mathrm{e}^{-\mathrm{i} \omega} \cdots \mathrm{e}^{-\mathrm{i} N \omega}\right]^{\mathrm{t}}$.

Since $h(n)$ is real valued, the frequency response $H(\omega)$ is conjugate symmetric, i.e.,

$H(-\omega)=H^{*}(\omega)$.

For the fractional differentiator design, the ideal frequency response $D(\omega)$ is given by

$D(\omega)=(\mathrm{i} \omega)^{v}= \begin{cases}|\omega|^{v} \mathrm{e}^{\mathrm{i} v \pi / 2}, & 0<\omega<\pi, \\ 0, & \omega=0, \\ |\omega|^{v} \mathrm{e}^{-\mathrm{i} v \pi / 2}, & -\pi<\omega<0 .\end{cases}$
Because the FIR filter $H(z)$ will introduce a delay and $D(\omega)$ is a zero delay specification, the FIR filter cannot be used to approximate the ideal respone $D(\omega)$. To solve this problem, we let the FIR filter approximate the modified specification $F_{\mathrm{d}}(\omega)$ which is the frequency response by cascading the ideal fractional differentiator and a pure delay system, i.e.,

$F_{\mathrm{d}}(\omega)=D(\omega) \mathrm{e}^{-\mathrm{i} n_{0} \omega}$,

where $n_{0}$ is a prescribed delay. Once filter $H(\omega)$ is designed, we use the following formula to obtain the design result $\hat{D}(\omega)$ of the fractional differentiator:

$\hat{D}(\omega)=H(\omega) \mathrm{e}^{\mathrm{i} n_{0} \omega}$.

Moreover, when a signal pass through filter $H(z)$, it is equivalent to passing through a cascade system of a pure delay filter $z^{-n_{0}}$ and a designed fractional differentiator with frequency response $(i \omega)^{v}$. In this paper, the filter coefficients $\boldsymbol{h}$ are obtained by minimizing the following least-squares error:

$J(\boldsymbol{h})=\int_{\omega \in\left(\boldsymbol{R}^{+} \cup \boldsymbol{R}^{-}\right)}\left|H(\omega)-F_{\mathrm{d}}(\omega)\right|^{2} \mathrm{~d} \omega$,

where frequency bands $R^{+}=[\alpha \pi, \pi]$ and $R^{-}=$ $[-\pi,-\alpha \pi]$. Note that $\alpha$ is a small positive number. Since the phase response jumps from $-v \pi / 2$ to $v \pi / 2$ at $\omega=0$, the interval $(-\alpha \pi, \alpha \pi)$ is a "don't care" region in the design process. Using the conjugate symmetric property of $H(\omega)$ and $F_{\mathrm{d}}(\omega)$, the error $J(\boldsymbol{h})$ can be rewritten in quadratic form:

$K(\boldsymbol{h})=\boldsymbol{h}^{\mathrm{t}} \boldsymbol{Q h}-2 \boldsymbol{h}^{\mathrm{t}} \boldsymbol{p}+c$,

where matrix $\boldsymbol{Q}$, vector $\boldsymbol{p}$, and scalar $c$ are real and given by

$\boldsymbol{Q}=2 \int_{\omega \in \boldsymbol{R}^{+}} \operatorname{Re}\left(\boldsymbol{e}(\omega) \boldsymbol{e}^{\mathrm{H}}(\omega)\right) \mathrm{d} \omega$,

$\boldsymbol{p}=2 \int_{\omega \in \boldsymbol{R}^{+}} \operatorname{Re}\left(F_{\mathrm{d}}(\omega) \boldsymbol{e}^{*}(\omega)\right) \mathrm{d} \omega$,

$c=2 \int_{\omega \in R^{+}}\left|F_{\mathrm{d}}(\omega)\right|^{2} \mathrm{~d} \omega$.

$\mathrm{H}$ denotes the Hermitian conjugate transpose operator, and $\operatorname{Re}(\cdot)$ stands for the real part of a complex 

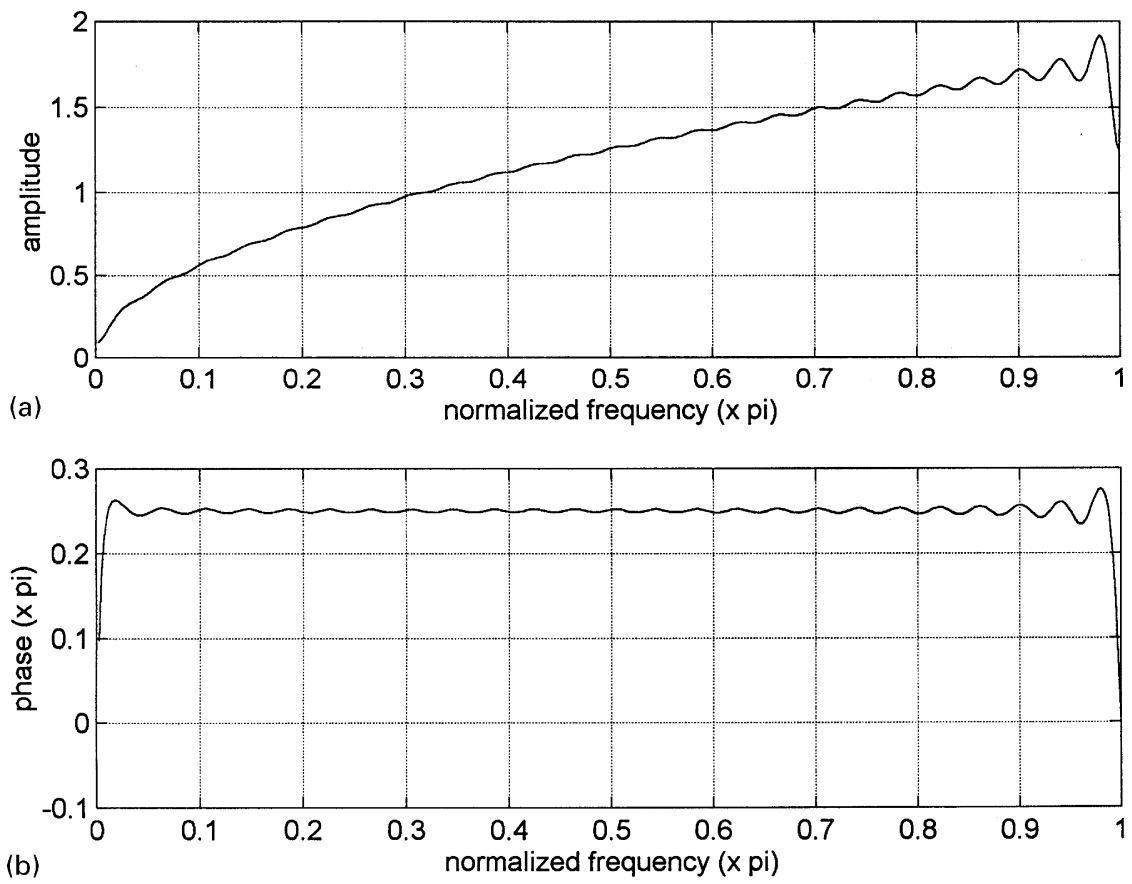

Fig. 2. The design result $H(\omega) \mathrm{e}^{\mathrm{i} n_{0} \omega}$ of a fractional order differentiator with $N=100, v=0.5$, and $n_{0}=50$. (a) amplitude response, (b) phase response.

number. Thus, the optimal solution of this problem is given by

$\boldsymbol{h}_{\mathrm{opt}}=\boldsymbol{Q}^{-1} \boldsymbol{p}$.

\subsection{Computation of fractional derivatives using the digital differentiator}

When a signal passes through the fractional order differentiator, the output will be its fractional derivative. Let us first design a differentiator with the prescribed fractional order. The parameters chosen are $N=100, v=0.5, n_{0}=50$, and $\alpha=0.02$. The design result $\hat{D}(\omega)=H(\omega) \mathrm{e}^{\mathrm{i} n_{0} \omega}$ is shown in Fig. 2. It is clear that the amplitude response approximates the specificaion $\sqrt{\omega}$ well. Also, the phase response fits $\pi / 4$ well for all frequencies except edge frequecies $\omega=0$ and $\pi$. Next, we use the designed fractional differentiator to filter the periodic rectangle sequence $x(n)$ defined by

$x(n)= \begin{cases}V, & k T \leqslant n<\left(k+\frac{1}{2}\right) T, \\ -V, & \left(k+\frac{1}{2}\right) T \leqslant n<(k+1) T,\end{cases}$ where $k$ is integer and $T$ is period. Choosing parameters $V=1$ and $T=100$, Fig. 3 depicts the fractional derivatives $D^{v} x(n)$ for various order $v$. It is clear that the results are very similar to those of continuous case shown in Fig. 1.

\section{Fractal process generation}

Since the fractional derivative of a zero mean white noise process is a fractal process called Brownian motion (fBm), the proposed method can be used to generate the fractal process. And, the better method to compute the fractional derivatives of signals, the better fractal process can be obtained. In this section, the designed fractional order differentiator is used to generate a fractal process which is better than the process obtained by the conventional method [3]. The spectrum of Brownian motion ( $\mathrm{fBm}$ ) is of the form $\omega^{v}$, where $v$ is a negative fractional number and $\omega$ is the frequency. Usually, the $\mathrm{fBm}$ is modeled as the output $y(n)$ of a rational filter $G(z)$ driven by a Gaussian 
(a)

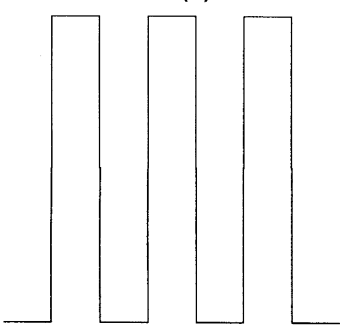

(c)
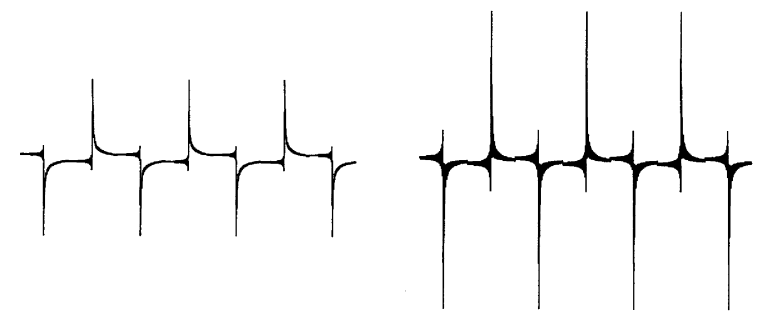

Fig. 3. The outputs of fractional order differentiator with various order $v$. (a) $v=0$, (b) $v=0.2$, (c) $v=0.5$, (d) $v=0.7$.

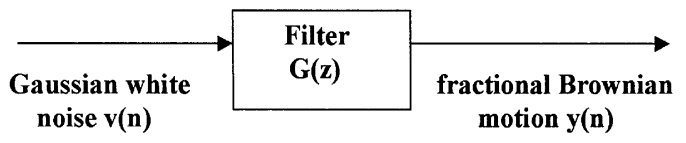

Fig. 4. A model to generate the fractional Brownian motion (fBm).

white noise $v(n)$ with zero mean and unit variance, as shown in Fig. 4. In [3], the transfer function of $G(z)$ is chosen as

$$
\begin{aligned}
G(z) & =\frac{1}{\left(1-z^{-1}\right)^{-v}} \\
& =\sum_{k=0}^{\infty}(-1)^{k+1} \frac{v(1-v) \cdots(k-1-v)}{k !},
\end{aligned}
$$

where $v$ is in the range $(-0.5,0.5)$. Hence, the $\mathrm{fBm}$ can be generated by the following difference equation:

$$
y(n)=\sum_{k=0}^{N}(-1)^{k+1} \frac{v(1-v) \cdots(k-1-v)}{k !} v(n-k),
$$

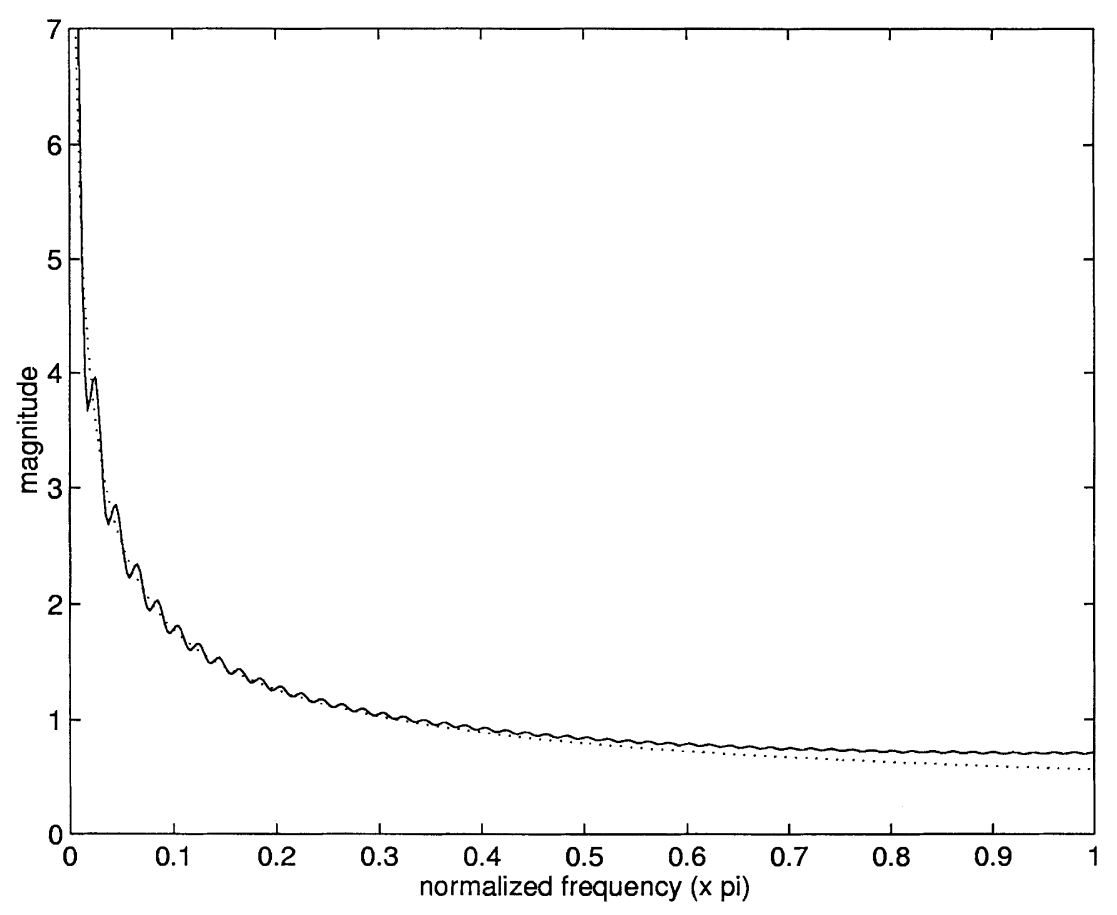

Fig. 5. The spectrum $\left|G\left(\mathrm{e}^{\mathrm{j} \omega}\right)\right|^{2}$ of filter $G(z)$ (solid line) and the ideal spectrum $\omega^{2 v}$ (dotted line). 


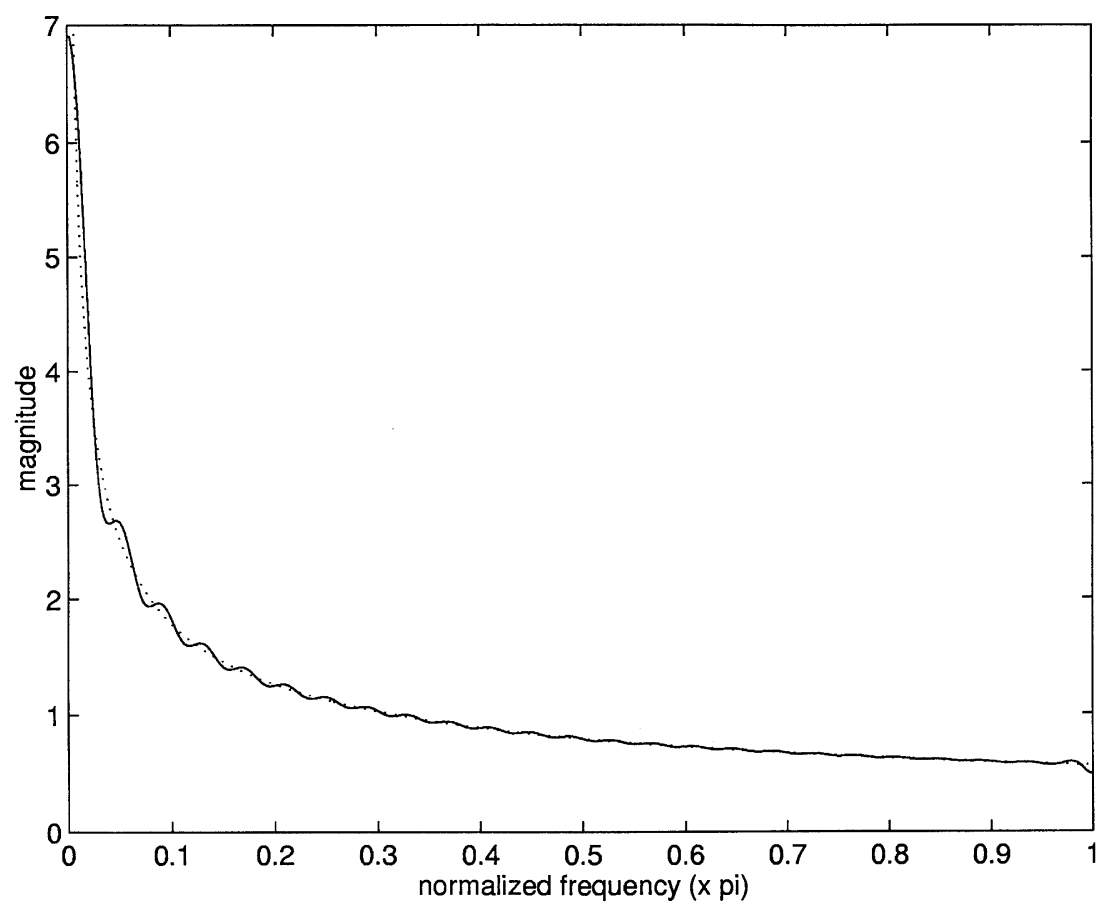

Fig. 6. The spectrum $\left|H\left(\mathrm{e}^{\mathrm{j} \omega}\right)\right|^{2}$ of filter $G(z)$ (solid line) and the ideal spectrum $\omega^{2 v}$ (dotted line).

where $N$ is the filter order of $G(z)$. The frequency response of filter $G(z)$ is given by

$G\left(\mathrm{e}^{\mathrm{i} \omega}\right)=\frac{1}{\left(1-\mathrm{e}^{-\mathrm{i} \omega}\right)^{-v}}=\left(2 \sin \left(\frac{\omega}{2}\right)\right)^{v} \mathrm{e}^{-\mathrm{i} \omega \nu / 2}$.

Since $\sin (\omega / 2)$ can be approximated as $\omega / 2$ when $\omega$ tends to zero, we have

$G\left(\mathrm{e}^{\mathrm{j} \omega}\right) \approx \omega^{v} \mathrm{e}^{-\mathrm{i} \omega v / 2}$

for small value of frequency $\omega$. This means that the spectrum $S_{y}(\omega)$ of fractal process $y(n)$ is

$S_{y}(\omega)=\left|G\left(\mathrm{e}^{\mathrm{i} \omega}\right)\right|^{2} \approx \omega^{2 v}$.

Thus, the spectrum behaves as $\omega^{2 v}$ as $\omega$ tends to zero. That is, at low frequency the spectrum of the process $y(n)$ has a behavior similar to that of the $\mathrm{fBm}$. However, at high frequencies, the spectrum of $y(n)$ will be far from that of fBm. Fig. 5 shows the spectrums with $v=-0.25$ and filter order $N=100$ to illustrate this fact. In order to get better model fitting, the filter $G(z)$ is replaced by the pro- posed digital differentiator $H(z)$ with negative fractional order. In this case, the spectrum $S_{y}(\omega)$ of fractal process $y(n)$ is given by

$S_{y}=\left|H\left(\mathrm{e}^{\mathrm{i} \omega}\right)\right|^{2}$.

As an example, a digital differentiator $H(z)$ with parameters $\quad N=100, v=-0.25, n_{0}=50$ and $\alpha=0.01$ is designed and chosen as the filter $G(z)$ in Fig. 4 to generate the fractal process $y(n)$. Fig. 6shows the spectrums of $\left|H\left(\mathrm{e}^{\mathrm{i} \omega}\right)\right|^{2}$ and ideal spectrum $\omega^{2 v}$. It is clear that the two curves are almost overlapped for all frequency. For comparison, an error measure is defined as follows:

$E=\left.\int_{0}^{\pi}\left|\omega^{2 v}-\right| G\left(\mathrm{e}^{\mathrm{i} \omega}\right)\right|^{2} \mid \mathrm{d} \omega$.

It is clear that the error $E$ depends on the choice of $G(z)$. Under the condition of the same filter order $N$, the error $E$ in Fig. 5 is 0.0809 which is greater than the error 0.0421 in Fig. 6. Thus, the proposed filter $G(z)$ generate a more accurate $\mathrm{fBm}$ than the 
conventional filter in [3]. The reason is that our filter is designed by an optimization method, whereas the filter in [3] is a heuristic one.

\section{Conclusion}

In this paper, the computation of fractional derivative using the Fourier transform and the digital FIR differentiator has been investigated. First, the Cauchy integral formula was generalized to define the fractional derivative of functions. Then, the fractional differentiation property of the Fourier transform of functions was presented. Using this property, the fractional derivative of function was computed in the frequency domain. Next, we developed a least-squares method to design a fractional order digital differentiator. When a signal passes through the designed differentiator, the output will be its fractional derivative. A design example is included to illustrate the effectiveness of this approach. Finally, the designed fractional order differentiator is used to generate a random fractal process which is better than the process obtained by the conventional method.

In future work, it will be interesting to apply fractional differentiators to any applications which use the computation of velocity and acceleration. For example, fractional differentiators can improve the success rate of online Chinese signature verification.

\section{References}

[1] L.B. Almeida, The fractional Fourier transform and time frequency representation, IEEE Trans. Signal Process. 42 (November 1994) 3084-3091.

[2] M.F. Barnsley, Fractal Everywhere, Academic, Boston, 1988.

[3] M. Deriche, A.H. Tewfik, Signal modeling with filtered fractional noise processes, IEEE Trans. Signal Process. 41 (9) (September 1993) 2839-2849.

[4] S.C. Dutta Roy, B. Kumar, Digital differentiators, in: N.K. Bose, C.R. Rao (Eds.), Handbook of Statistics, Vol. 10, Elsevier Science Publishers, Amsterdam, 1993, pp. 159-205.

[5] N. Engheta, On the role of fractional calculus in electromagnetic theory, IEEE Antennas Propagation Mag. 39 (4) (August 1997) 35-46.
[6] A. Fenander, A fractional derivative railpad model included in a railway track model, J. Sound Vib. 212 (5) (1998) pp. 889-903.

[7] J. Lancis, T. Saoplik, E. Tajahuerce, V. Climent, M. Fernandez-Alonso, Fractional derivative Fourier plane filter for phase-change visualization, Appl. Opt. 36 (29) (October 1997) 7461-7464.

[8] B. Mbodje, G. Montseny, Boundary fractional derivative control of the wave equation, IEEE Trans. Automat. Control 40 (2) (February 1995) 378-382.

[9] K.S. Miller, B. Ross, An Introduction to the Fractional Calculus and Fractional Differential Equations, Wiley, New York, 1993.

[10] V. Namias, The fractional order Fourier transform and its application to quantum mechanics, J. Inst. Maths Appl. 25 (1980) 241-265.

[11] K. Nishimoto, Nishimoto's fractional calculus of elementary functions, Proceedings of the International Conference of Fractonal Calculus and its Applications, College of Engineering, Nihon University, Tokyo, May-June, 1989, pp. 112-122.

[12] K.B. Oldham, J. Spanier, The Fractional Calculus, Academic Press, New York, 1974.

[13] A.V. Oppenheim, R.W. Schafer, Discrete-Time Signal Processing, Prentice-Hall, Englewood Cliffs, NJ, 1989.

[14] T.W. Parks, J.H. McClellan, Chebyshev approximation for non recursive digital filters with linear phase, IEEE Trans. Circuit Theory 19 (March 1972) 189-194.

[15] S.C. Pei, J.J. Shyu, Design of FIR Hilbert transformers and differentiators by eigenfilters, IEEE Trans. Circuits Systems 35 (November 1988) 1457-1461.

[16] S.C. Pei, J.J. Shyu, Eigenfilter design of higher-order digital differentiators, IEEE Trans. Acoust. Speech Signal Process. 37 (1989) 505-511.

[17] S.C. Pei, M.H. Yeh, C.C. Tseng, Discrete fractional Fourier transform based on othogonal projections, IEEE Trans. Signal Process. 47 (May 1999) 1335-1348.

[18] L.A. Rubenfeld, A First Course in Applied Complex Variables, Wiley, New York, 1985.

[19] M. Shao, C.L. Nikias, Signal processing with fractional lower order moment: stable processes and their applications, Proc. IEEE 81 (July 1993) 986-1010.

[20] H. Shekarforous, J. Zerubia, M. Berthod, Denoising by extracting fractional order singularities, ICASSP 98, pp. 2889-2892.

[21] M.I. Skolnik, Introduction to Radar Systems, McGrawHill, New York, 1980.

[22] S. Sunder, W.S. Lu, A. Antoniou, Y. Su, Design of digital differentiators satisfying prescribed specifications using optimization techniques, Proc. Inst. Elect. Eng. Pt. G 138 (June 1991) 315-320.

[23] S. Sunder, V. Ramachandra, Design of equiripple nonrecursive digital differentiators and Hilbert transformers using a weighted least-squares technique, IEEE Trans. Signal Processing 42 (9) (September 1994) 2504-2509.

[24] S. Usui, I. Amidror, Digital lowpass differentiation for biological signal processing, IEEE Trans. Biomedical Eng. 29 (1982) 686-693. 